August 15-18, 2010, Montreal, Quebec, Canada

\title{
DETC2010-28829
}

\section{COMPLETELY ELIMINATING THE SINGULARITIES OF A 3-DOF PLANAR PARALLEL ROBOT WITH ONLY ONE DEGREE OF ACTUATION REDUNDANCY}

\author{
Novona Rakotomanga \\ Ilian A. Bonev \\ Department of Automated Manufacturing Engineering \\ École de technologie supérieure (ÉTS), Montreal, QC, Canada \\ ilian.bonev@etsmtl.ca
}

\begin{abstract}
The Cartesian workspace of an $n$-DOF parallel robot $(n<6)$ is generally divided by singularity hyper-surfaces of dimension $n-1$. A common approach to reducing the dimension of the singularity manifold is to use actuation redundancy. However, in all previously reported works, adding one redundant actuator reduces the dimension of the singularity manifold by only one. This paper is the first to demonstrate that a properly designed actuation redundancy can be much more effective than this. Specifically, a $3-\underline{R} P R$ design is presented in which the mobile platform and the base are equilateral triangles and show that adding a single $\underline{R} P R$ leg connecting the centers of these two triangles completely eliminates the singularities of the robot, which are otherwise a surface in the Cartesian space.
\end{abstract}

\section{INTRODUCTION}

Most parallel robots have singularities that limit their workspace. The less harmful ones occur when one leg is at a singularity, or, in other words, when the mobile platform looses one or more degrees of freedom (DOF). We loosely call these Type 1 singularities (other authors call them serial singularities). The most dangerous singularities, though, are those associated with the loss of stiffness of the mobile platform, which we loosely call Type 2 singularities (sometimes also referred to as parallel singularities or force-unconstrained configurations). Indeed, approaching a Type 2 singularity often requires large actuator torques or forces. Hence, these singularities should be avoided for most applications. A safe solution is to eliminate Type 2 singularities at the very design stage through optimization $[1,2]$, or to determine singularityfree zones in the workspace [3] and limit the motion of the mobile platform through careful trajectory planning.

\footnotetext{
${ }^{1}$ It is customary to refer to parallel mechanisms using the symbols P and R, which stand for prismatic and revolute joints respectively. When a joint is actuated, its symbol is underlined.
}

For an $n$-DOF parallel robot, the Type 2 singularity loci in the Cartesian space generally constitute an $n-1$ dimensional entity. For example, the singularity loci of a general 3-DOF 3- $R R R^{1}$ spherical parallel robot constitute a two-dimensional surface separating the three-dimensional (orientation) workspace into aspects. In [4], however, it was demonstrated that for a 3- $\underline{R} R R$ parallel wrist in which all mutually fixed axes are normal, a design known as the Agile Eye, Type 2 singularity surfaces degenerate into curves.

Reducing the dimension of the Type 2 singularity manifold is, however, generally done through redundant actuation, i.e., adding more actuators than the number of DOFs of the robot, by either actuating some of the passive joints or by adding actuated legs [5-8]. To the best of our knowledge, in all dozens of reported works, each redundant actuator reduces the dimension of the Type 2 singularity manifold by only one. (It is interesting to note that in [9], the authors actually studied the design conditions for which an additional actuated leg does not modify the singularity manifold at all.) Thus, for example, in order to completely eliminate the Type 2 singularities of a planar 3-DOF parallel robot, one would need to add three actuators [10]!

We therefore believe that this paper is the first to report the possibility of eliminating all Type 2 singularities of a particular planar 3-DOF parallel robot through the addition of only one degree of actuation redundancy. Specifically, we present a 3- $\underline{R} P R$ design in which the mobile platform and the base are equilateral triangles and show that adding a single $\underline{R} P R$ leg connecting the centers of these two triangles completely eliminates the Type 2 singularities of the robot, which are otherwise a surface in the $x y \phi$ Cartesian space.

\section{INPUT-OUTPUT VELOCITY EQUATIONS}

Consider a 3- $\underline{R} P R$ planar parallel robot. We denote with $A_{i}$ and $B_{i}(i=1,2,3)$ the intersections of the base and platform 
revolute joint axes respectively, with a plane normal to these axes. Let points $A_{\mathrm{i}}$ form an equilateral triangle and so do points $B_{i}$. Then, let $O x y$ be the base reference frame, with $O$ being the center of the base triangle and the $x$ axis passing through $A_{1}$. Let also $C x^{\prime} y^{\prime}$ be the mobile reference frame, with $C$ being the center of the mobile platform triangle and the $x$, axis passing through $B_{1}$. Therefore, the only two design parameters of the parallel robot under study are the lengths of $O A_{i}$ and $C B_{i}$, which will be denoted by $a$ and $b$, respectively.

We define each active-joint variable $\theta_{i}$ as the angle between the $x$ axis and the positive direction of the prismatic joint of leg $i$, measured in the counter-clockwise sense. The distance from point $A_{i}$ to point $B_{i}$ along the positive direction of prismatic joint $i$ is denoted by $\rho_{i}$. Finally, the Cartesian coordinates of the mobile platform are the position of point $C$ with respect to $O x y$, denoted by $(x, y)$, and the orientation of $C x^{\prime} y^{\prime}$ with respect to $O x y$, denoted by $\phi$.

Now, let us add a fourth $\underline{R} P R$ leg, referred to as leg 0 , so that the axis of its actuated revolute joint passes through $O$ and the axis of the platform revolute joint passes through $C$, as shown in Fig. 1. The fourth active-joint variable will be denoted by $\theta_{0}$, while the directed distance between $O$ and $C$ will be denoted by $\rho_{0}$. From now on, $i=0,1,2,3$.

The resulting 4- $R P R$ 3-DOF planar parallel robot has therefore only one degree of actuation redundancy. For this mechanism to be in a Type 2 singularity, each of the four possible three-legged sub-mechanisms should be in a Type 2 singularity. Let us refer to the $3-\underline{R} P R$ mechanism that excludes leg $i$ as sub-mechanism $i$.

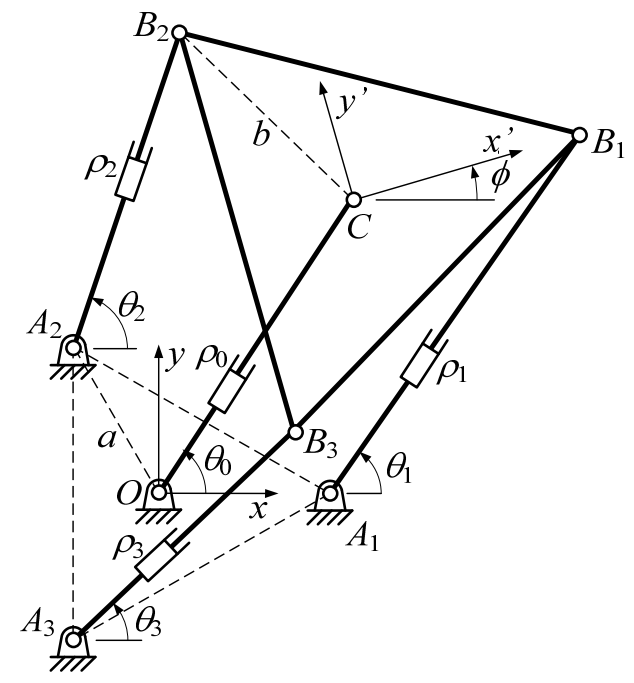

Fig. 1. The $4-\underline{R} P R$ planar parallel robot under study.

The constraint equations for each of the four legs, valid only when a leg is not singular $\left(\rho_{i} \neq 0\right)$, are as follows:

$$
\begin{aligned}
& x \sin \theta_{0}-y \cos \theta_{0}=0, \\
& (x+b \cos \phi-a) \sin \theta_{1}-(y+b \sin \phi) \cos \theta_{1}=0, \\
& (x+b \cos (\phi+2 \pi / 3)-a \cos (2 \pi / 3)) \sin \theta_{2} \\
& \quad-(y+b \sin (\phi+2 \pi / 3)-a \sin (2 \pi / 3)) \cos \theta_{2}=0, \\
& (x+b \cos (\phi+4 \pi / 3)-a \cos (4 \pi / 3)) \sin \theta_{3} \\
& \quad-(y+b \sin (\phi+4 \pi / 3)-a \sin (4 \pi / 3)) \cos \theta_{3}=0 .
\end{aligned}
$$

The active-joint variables $\theta_{i}$ are easy to obtain from these four equations. Differentiating the latter with respect to time yields the input-output velocity equations of our parallel mechanism. For each of the four possible three-legged submechanisms, we have

$$
\mathbf{A}_{\mathrm{SMi}} \mathrm{t}=\mathbf{B}_{\mathrm{SMi}} \dot{\mathrm{q}}_{\mathrm{SMi}}
$$

where $\mathrm{t}=[\dot{x}, \dot{y}, \dot{\phi}]^{T}, \dot{\mathrm{q}}_{\mathrm{SMi}}$ is the vector of active-joint rates for sub-mechanism $i$, and $\mathbf{A}_{\mathrm{SM} i}$ and $\mathbf{B}_{\mathrm{SM} i}$ are the Jacobian matrices for sub-mechanism $i$. The latter are defined as:

$$
\begin{aligned}
\mathbf{A}_{\mathrm{SMi}} & =\left[\begin{array}{lll}
\sin \theta_{j} & -\cos \theta_{j} & M_{j} \\
\sin \theta_{k} & -\cos \theta_{k} & M_{k} \\
\sin \theta_{l} & -\cos \theta_{l} & M_{l}
\end{array}\right] \\
\mathbf{B}_{\mathrm{SMi}} & =\operatorname{diag}\left(\rho_{j}, \rho_{k}, \rho_{l}\right)
\end{aligned}
$$

with $i \neq j \neq k \neq l, j, k$ and $l=0,1,2,3$

and

$$
\begin{aligned}
& M_{0}=0 \\
& M_{1}=-b\left(\sin \phi \sin \theta_{1}+\cos \phi \cos \theta_{1}\right) \\
& M_{2}=-b\left(\sin (\phi+2 \pi / 3) \sin \theta_{2}+\cos (\phi+2 \pi / 3) \cos \theta_{2}\right) \\
& M_{3}=-b\left(\sin (\phi+4 \pi / 3) \sin \theta_{3}+\cos (\phi+4 \pi / 3) \cos \theta_{3}\right)
\end{aligned}
$$

As it is well known, there is a Type 1 singularity if and only if $\operatorname{det}\left(\mathbf{B}_{\mathrm{SMi}}\right)=0$. For each sub-mechanism $i$, a Type 2 singularity occurs if and only if $\operatorname{det}\left(\mathbf{A}_{\mathrm{SMi}}\right)=0$.

\section{TYPE 2 SINGULARITY LOCI FOR EACH THREE- LEGGED SUB-MECHANISM}

To obtain the expressions for the Type 2 singularity loci in the $x y \phi$ Cartesian space for each sub-mechanism $i$, we simply calculate the determinants of matrices $\mathbf{A}_{\mathrm{SM} i}$ and perform variable substitutions using the inverse kinematic equations, obtained easily from Eqs. (1-4). Note, however, that the resulting equations are valid only when none of the legs is in a singularity $\left(\rho_{i} \neq 0\right)$.

There are two important cases that must be distinguished. The Type 2 singularity equations in the $x y \phi$ Cartesian space for each sub-mechanism $i$ are given as follows.

\subsection{Case 1: $(b-a \cos \phi) \neq 0$}

Type 2 singularity curve for sub-mechanism 0 is obtained by calculating the determinant of matrix $\mathbf{A}_{\mathrm{SM}}$ :

$$
x^{2}+y^{2}-\left(a^{2}+b^{2}-2 a b \cos \phi\right)=0 \text {. }
$$

For a given orientation, $\phi$, the above equation represents a circle centered at $\mathbf{p}_{0}$ and of radius $r$, where

$$
\mathbf{p}_{0}=\left[\begin{array}{l}
0 \\
0
\end{array}\right] \text { and } r=\sqrt{a^{2}+b^{2}-2 a b \cos \phi} .
$$

Note that the expression under the square root in $r$ is always positive and non-zero. 
Type 2 singularity curve for sub-mechanism 1 is defined as:

$$
x^{2}+y^{2}-2(b \cos \phi-a) x-2 b \sin \phi y=0 \text {. }
$$

For a given orientation, $\phi$, the above equation represents a circle centered at $\mathbf{p}_{1}$ and of radius $r$, where

$$
\mathbf{p}_{1}=\left[\begin{array}{c}
b \cos \phi-a \\
b \sin \phi
\end{array}\right] .
$$

Type 2 singularity curve for sub-mechanism 2 is defined as:

$$
\begin{array}{r}
x^{2}+y^{2}-(-(b \cos \phi-a)-\sqrt{3} b \sin \phi) x \\
-(\sqrt{3}(b \cos \phi-a)-b \sin \phi) y=0 .
\end{array}
$$

For a given orientation, $\phi$, the above equation represents a circle centered at $\mathbf{p}_{2}$ and of radius $r$, where

$$
p_{2}=\left[\begin{array}{l}
-\frac{1}{2}(b \cos \phi-a)-\frac{\sqrt{3}}{2} b \sin \phi \\
\frac{\sqrt{3}}{2}(b \cos \phi-a)-\frac{1}{2} b \sin \phi
\end{array}\right] .
$$

Type 2 singularity curve for sub-mechanism 3 is defined as:

$$
\begin{aligned}
x^{2}+y^{2} & -((b \cos \phi-a)+\sqrt{3} b \sin \phi) x \\
& -(-\sqrt{3}(b \cos \phi-a)-b \sin \phi) y=0 .
\end{aligned}
$$

For a given orientation, $\phi$, the above equation represents a circle centered at $\mathbf{p}_{3}$ and of radius $r$, where

$$
\mathbf{p}_{3}=\left[\begin{array}{c}
-\frac{1}{2}(b \cos \phi-a)+\frac{\sqrt{3}}{2} b \sin \phi \\
-\frac{\sqrt{3}}{2}(b \cos \phi-a)-\frac{1}{2} b \sin \phi
\end{array}\right] .
$$

\subsection{Case 2: $(b-a \cos \phi)=0$}

In this case, all four sub-mechanisms are at a Type 2 singularity. In the $x y \phi$ Cartesian space, these singularities correspond to two planes parallel to the $x y$ plane.

\section{TYPE 2 SINGULARITIES DESAPPEAR}

In a general $4-\underline{R} P R$ planar parallel robot, a Type 2 singularity occurs when each of the four possible three-legged submechanisms is in a Type 2 singularity. In other words, a point (a pose) in the Cartesian space corresponds to a Type 2 singularity of the redundant robot if it belongs to each of the four three-legged mechanism singularity surfaces. For example, Fig. 2 shows the Type 2 singularity surface in the $x y \phi$ Cartesian space of the initial 3- $\underline{R} P R$ planar parallel robot (i.e., submechanism 0 , composed of legs 1,2 and 3).

These four singularity surfaces generally intersect at one or more curves. For example, Fig. 3 shows the Type 2 singularity loci (two curves shown in different colours and intersecting at one point) in the $x y \phi$ Cartesian space of a $4-\underline{R} P R$ planar parallel robot, in which $A_{1} A_{2} A_{3}$ and $B_{1} B_{2} B_{3}$ form equilateral triangles, but $A_{0}$ and $B_{0}$ (the anchor points of the redundant leg) are at some arbitrary positions.

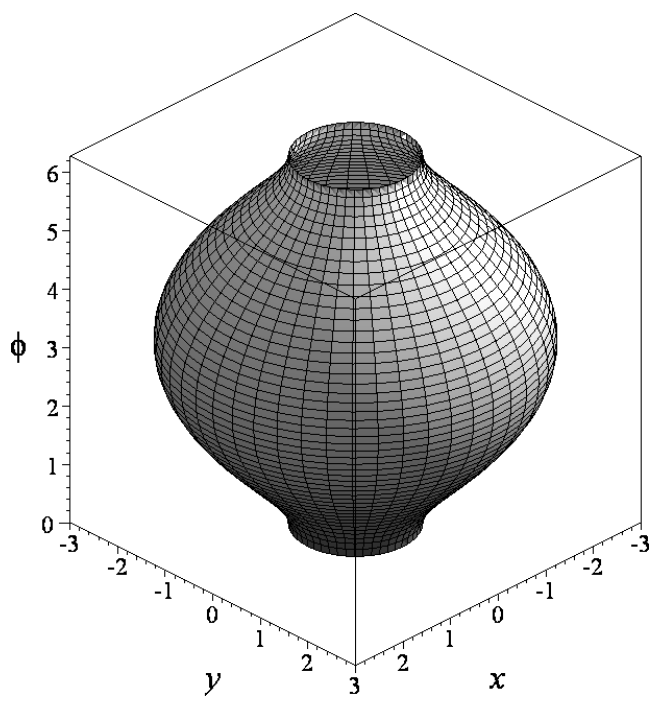

Fig. 2. Singularity loci of a $3-\underline{R} P R$ planar parallel robot.

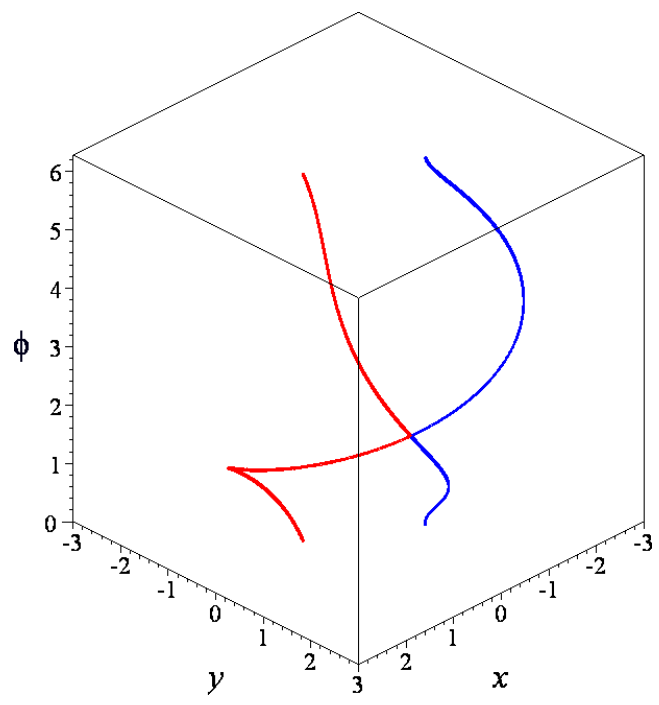

Fig. 3. Singularity loci of a general $4-\underline{R} P R$ planar parallel robot.

As we saw in the previous section, the zeroing of the expression $(b-a \cos \phi)$ makes all the difference for the Type 2 singularity loci. Studying this condition will help us optimize our design.

\subsection{Case 1: $a<b$}

In this case, the expression $(b-a \cos \phi)$ is always different than zero, whatever the orientation $\phi$. Therefore, as we saw in Section 3.1, the Type 2 singularity loci of each submechanism represent a circle in the plane $x y$, whatever the orientation $\phi$. Note that these four circles have the same radius, which varies with the orientation $\phi$, but is never zero. Recall also that the circle for sub-mechanism 0 is centered at the origin $O$. The centers of the other three circles lie on the first circle, at equal distances from each other, as illustrated in Fig. 4.

Thus, it is obvious that the four circles never intersect at a common point. Therefore, the workspace of the redundant 4- $\underline{R} P R$ planar parallel robot under study is free of any Type 2 singularities. 

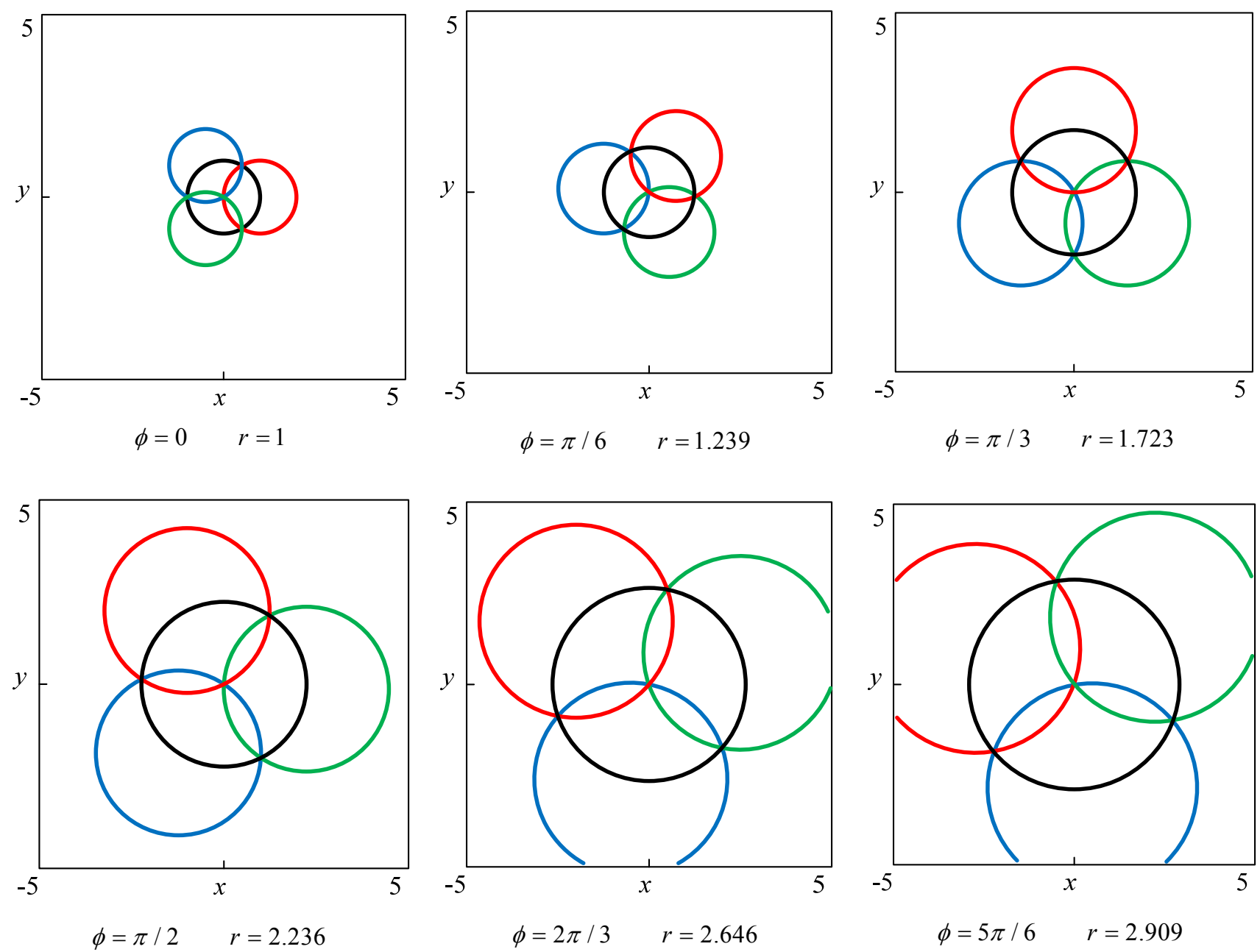

Fig. 4. Type 2 singularities for each of the four three-legged sub-mechanisms, for several values of $\phi(a=1, b=2)$.

\subsection{Case 2: $a \geq b$}

In this case, the expression $(b-a \cos \phi)$ can be zeroed at two values of $\phi$. In these two orientations, the redundant parallel robot under study is always at a Type 2 singularity, whatever the values of $x$ and $y$. Therefore, in this case, the redundant actuation does not reduce the dimension of the Type 2 singularity manifold.

\section{CONCLUSIONS}

To the best of our knowledge, this is the first demonstration of how a single degree of actuation redundancy can eliminate all Type 2 singularities of a 3-DOF planar parallel robot. In particular, it was shown that the proper design of a redundant $R P R$ leg added between the centers of the base and mobile platform of a symmetric 3- $\underline{R} P R$ planar parallel robot eliminates all Type 2 singularities. It was also shown that this is true only if the base equilateral triangle is smaller than the mobile platform equilateral triangle. In the opposite case, there is no gain at all in terms of singularities.

\section{REFERENCES}

[1] Jiang, Q., and Gosselin, C.M., "Geometric optimization of planar 3-RPR parallel mechanisms," Transactions of the Canadian Society for Mechanical Engineers, Vol. 31, No. 4, pp. 457-468, 2007.

[2] Arsenault, M., and Boudreau, R., "The synthesis of three-degree-of-freedom planar parallel mechanisms with revolute joints (3-RRR) for an optimal singularity free workspace," Journal of Robotic Systems, Vol. 21, No. 5, pp. 259-274, 2004.

[3] Li, H., and Gosselin, C.M., "Determination of maximal singularity-free zones in the workspace of planar threedegree-of-freedom parallel mechanisms," Mechanism and Machine Theory, Vol. 41, No. 10, pp. 1157-1167, 2006.

[4] Bonev, I.A., Chablat, D., and Wenger, P., "Working and assembly modes of the Agile Eye," IEEE International Conference on Robotics and Automation, Orlando, FL, USA, pp. 2317-2322, May 15-19, 2006.

[5] Notash, L., and Podhorodeski, R.P., "Forward displacement analysis and uncertainty configurations of parallel manipulators with a redundant branch," Journal of Robotic Systems, Vol. 13, No. 9, pp. 587-601, 1996.

[6] Dasgupta, B., and Mruthyunjaya, T.S., "Force redundancy in parallel manipulators: theoretical and practical issues," Mechanisms and Machine Theory, Vol. 33, No. 6, pp. 727-742, 1998. 
[7] Choudhury, P., and Ghosal, A., "Singularity and controllability analysis of parallel manipulators and closed-loop mechanisms," Mechanism and Machine Theory, Vol. 35, No. 10, pp. 1455-1479, 2000.

[8] Firmani, F., and Podhorodeski, R.P., "Forceunconstrained poses of the 3-PRR and 4-PRR planar parallel manipulators," Transactions of the Canadian Society for Mechanical Engineering, Vol. 29, No. 4, pp. 617-628, 2005.

[9] Husty, M., and Mielczarek, S., and Hiller, M., "Constructing an overconstrained planar 4RPR manipulator with maximal forward kinematics solution set," Proceedings of the l0th International Workshop on Robotics in the Alpe-Adria-Danube Region, Vienna, Austria, 2001.

[10] Firmani, F., and Podhorodeski, R.P., "Forceunconstrained poses for a redundantly-actuated planar parallel manipulator," Mechanism and Machine Theory, Vol. 39, No. 5, pp. 459-476, 2004. 